\title{
Extending parton branching TMDs to small $x$
}

\section{Sara Taheri Monfared*}

DESY, Hamburg, FRG

E-mail: sara.taheri.monfarededesy.de

\section{Francesco Hautmann}

Elementary Particle Physics, University of Antwerp, B 2020 Antwerp

RAL, Chilton OX11 OQX and University of Oxford, Oxford OX1 3NP

UPV/EHU, University of the Basque Country, E48080 Bilbao

E-mail: hautmann@thphys.ox.ac.uk

\section{Hannes Jung}

DESY, Hamburg, FRG

E-mail: hannes.jung@desy . de

Melanie Schmitz

DESY, Hamburg, FRG

E-mail: melanie.schmitz@desy.de

\begin{abstract}
We explore the possibility to include small- $x$ dynamics effects in the parton branching (PB) approach to transverse momentum dependent (TMD) parton distribution functions. To this end, we first revisit the PB method at leading order, presenting a new fit to inclusive-DIS precision data, and performing a numerical study of the dynamic soft-gluon resolution scale. Next we investigate the effects of modified CCFM kernels, including both Sudakov and non-Sudakov form factors.
\end{abstract}

XXVII International Workshop on Deep-Inelastic Scattering and Related Subjects - DIS2019 8-12 April, 2019

Torino, Italy

${ }^{*}$ Speaker. 


\section{Introduction}

In Refs. [1,2] a parton branching (PB) equation has been proposed for the evolution of transverse momentum dependent (TMD) parton distribution functions. In this approach, soft gluon emission and transverse momentum recoils in the QCD evolution are treated by introducing the soft-gluon resolution scale $z_{M}$ to separate resolvable and non-resolvable branchings, and the Sudakov form factors $\Delta_{a}$ (where $a=1, \ldots, 2 N_{f}+1$ is the flavor index, with $N_{f}$ the number of quark flavors) to express the probability for no resolvable branching in a given evolution interval.

The purpose of the study reported in this article is to extend the PB TMD approach to include effects of small- $x$ dynamics. The study consists of two parts: a numerical analysis of the PB method at leading order (LO) in the strong coupling $\alpha_{s}$, for which in particular we carry out a detailed investigation of the role of the soft-gluon resolution scale $z_{M}$; an extension of the PB evolution kernels to the small $x$ region by including, besides the Sudakov form factors, also the non-Sudakov form factors according to CCFM methods $[3,4,5]$.

The article is organized as follows. In Sec. 2 we describe the LO PB analysis, performing a new fit to inclusive-DIS precision data [6]. In Sec. 3 we study the dependence of the fit on the parameter $q_{0}$, which controls the minimum transverse momentum emitted in the branching evolution and is related to the soft-gluon resolution scale $z_{M}$ according to angular ordering $[1,7]$. In Sec. 4 we introduce modified CCFM kernels and investigate their effect on the results of parton evolution. We give conclusions in Sec. 5 .

\section{PB method at LO}

The PB method has been developed in $[1,2]$ evaluating the evolution kernels up to nextto-leading order (NLO). These results have been used in Ref. [8] to perform NLO fits to precision HERA1+2 measurements [6] and in Ref. [9], along with NLO matrix elements for Drell-Yan hadroproduction, to make predictions for Z-boson transverse momentum spectra at the LHC.

Our goal in this work is to explore the extension of the PB method to include small- $x$ CCFM effects and for this purpose it is useful to start from the formulation of the PB method at LO. To this end, we have performed an analysis at LO analogous to that of Ref. [8] and obtained PB TMD fits at LO. These LO fits may be useful in their own right for further applications.

The fits are performed using the open-source fitting platform $x F$ itter [10] and the numerical techniques developed in $[11,12]$ to treat the transverse momentum dependence in the fitting procedure. Similarly to Ref. [8], we consider the two angular ordered TMD sets (Set1 and Set2) using two different choices for the $\alpha_{s}$ evolution. We consider the same functional forms for initial densities and proceed with the LO approximation for an extension towards the LO CCFM evolution. The integrated TMD is fitted within $\times F$ itter at LO, where the PB method is implemented, to precision HERA1+2 measurements in the range of $3.5<Q^{2}<50000 \mathrm{GeV}^{2}$ and $4.10^{-5}<x<0.65$ [6]. Our $\chi^{2} / d o f$ for both sets are reasonably good (1.24 and 1.26 for set 1 and set2, respectively) and comparable to the NLO fit.

\section{Soft-gluon resolution effects}

Partons emitted with a transverse momentum smaller than a certain value given by the resolu- 
tion scale cannot be resolved. The resolution scale $z_{M}$ can change dynamically with the evolution scale. For angular ordering condition we consider $z_{M}=1-\frac{q_{0}}{q_{i}}$, while $q_{0}$ is chosen to be very small $\left(q_{0}=0.01 \mathrm{GeV}\right)$ to ensure that the PB evolution coincides with the standard DGLAP evolution.

We perform fits to DIS data with different choices of $q_{0}$. In Fig. 1 we illustrate how much would be the actual distribution changed by varying $z_{M}$. We observe that with higher $q_{0}$ (smaller
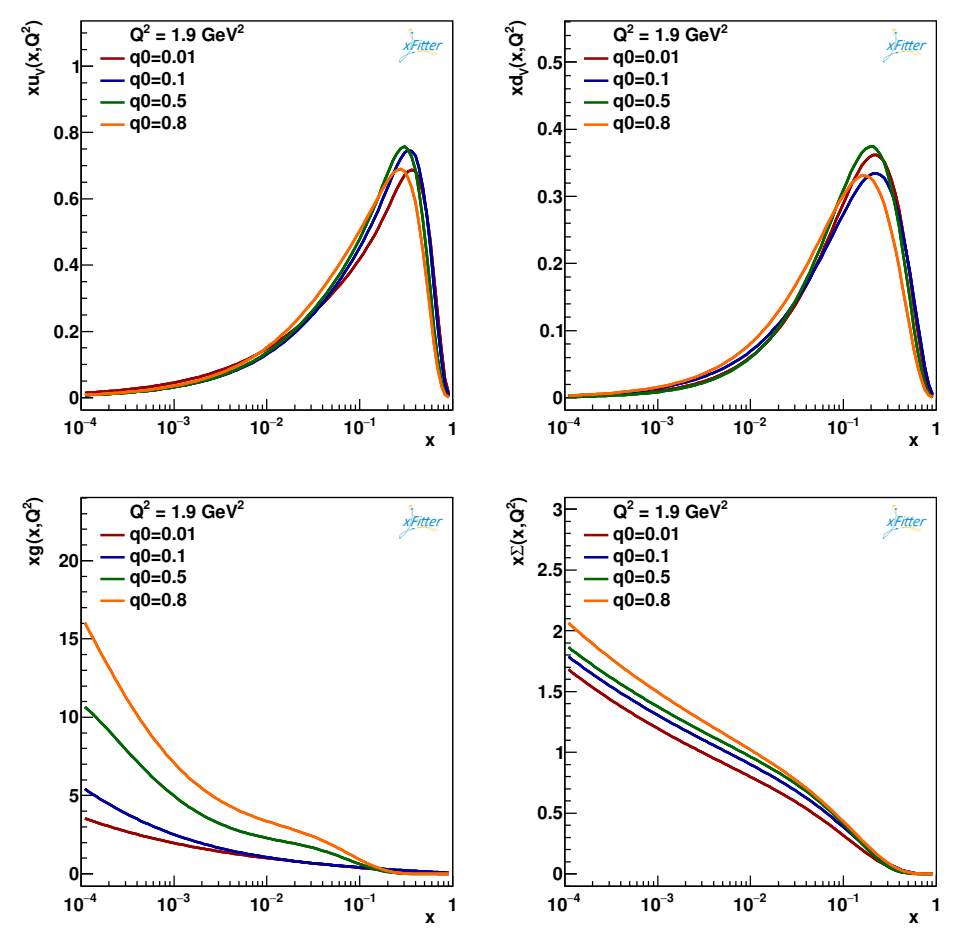

Figure 1: Parton densities for different values of $q_{0}$ at $Q^{2}=1.9 \mathrm{GeV}^{2}$

$z_{M}$ ), the gluon distribution becomes significantly larger, essentially in the small $x$ region and the $\chi^{2} /$ dof also increases.

We investigate further how the choice of $Q_{\min }^{2}$, the minimum $Q^{2}$ of the data within the fit, influences the quality of fit. We perform several fits with the different $Q_{\min }^{2}$. As illustrated in Fig 1, a reasonable fit can be determined for larger $q_{0}$ if $Q_{\min }^{2}>10 \mathrm{GeV}^{2}$.

\section{Extension to include small $x$ processes}

In this section we describe how the full angular ordering condition enlarges the phase space. We go from the DGLAP ordering $\left(q_{i}>q_{, i-1}\right)$ to the angular ordering $\left(q_{i}>z_{i-1} q_{i-1}\right)$. Here $q_{i}$ are the rescaled transverse momenta. In terms of the transverse momentum in the $t$-channel, DGLAP ordering means $k_{\perp}<q_{i}$, while angular ordering also covers $k_{\perp}>q_{i}$. This is due to the coherent effects. We need to introduce a non-Sudakov form factor to sum all virtual corrections in which the rescaled transverse momenta of on-shell emitted gluons are smaller than the $k_{\perp}\left(k_{\perp}>q_{i}\right)$.The 


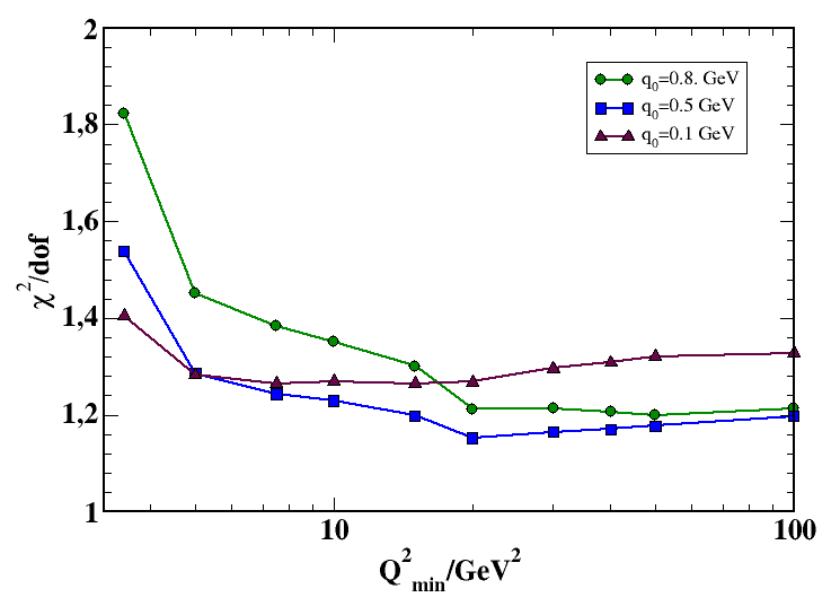

Figure 2: The dependence of $\chi^{2} / d o f$ on $Q_{\min }^{2}$ of LO fit for $q_{0}=0.1,0.5,0.8 \mathrm{GeV}$

modified CCFM splitting functions at LO including the non-Sudakov form factor are given by

$$
\begin{aligned}
P_{g g}^{(0)} & =6\left(\frac{\alpha_{s}}{2 \pi}\right)\left(\frac{1}{z} \widetilde{\Delta}_{n s}+\frac{1}{1-z}-2+z(1-z)\right) \widetilde{\Delta}_{s}, \\
P_{g q}^{(0)} & =\frac{4}{3}\left(\frac{\alpha_{s}}{2 \pi}\right)\left(z-2+\frac{2}{z} \widetilde{\Delta}_{n s}\right) \widetilde{\Delta}_{s}, \\
P_{q g}^{(0)} & =\frac{1}{2}\left(\frac{\alpha_{s}}{2 \pi}\right)\left(z^{2}+(1-z)^{2}\right) \widetilde{\Delta}_{s}, \\
P_{q_{i} q_{i}}^{(0)} & =\frac{4}{3}\left(\frac{\alpha_{s}}{2 \pi}\right)\left(\frac{1+z^{2}}{1-z}\right) \widetilde{\Delta}_{s} .
\end{aligned}
$$

with $\widetilde{\Delta}_{s}$ and $\widetilde{\Delta}_{n s}$ defined as

$$
\begin{aligned}
& \widetilde{\Delta}_{s}=\exp \left(-\int_{z_{i-1} q_{i-1}}^{q_{i}} \frac{d q^{\prime 2}}{q^{\prime 2}} \int^{z_{M}} d z \frac{1}{1-z}\right), \\
& \widetilde{\Delta}_{n s}=\exp \left(-\int_{z_{i-1} q_{i-1}}^{k_{\perp}} \frac{d q^{\prime 2}}{q^{\prime 2}} \int^{z_{M}} d z \frac{1}{z}\right) .
\end{aligned}
$$

The non-Sudakov form factor is relevant only when the emitted gluon is fast and therefore it is important only in presence of the $1 / z$ term in the DGLAP splitting function. Therefore we included them in $P_{g g}$ and $P_{g q}$ splitting functions.

In the next step, we re-write the CCFM splitting functions in terms of the full DGLAP splitting function with the DGLAP Sudakov form factor:

$$
\widetilde{\Delta}_{s} \rightarrow \Delta_{s}=\exp \left(-\int_{z_{i-1} q_{i-1}}^{q_{i}} \frac{d q^{\prime 2}}{q^{\prime 2}} \int^{z_{M}} d z\left(\frac{1}{z}+\frac{1}{1-z}-2+z(1-z)\right)\right) .
$$

We observe that $\frac{1}{z} \Delta_{n s} \Delta_{s}$ is already covered by $\frac{1}{z} \Delta_{s}$ for $k_{\perp}<q_{i}$ and the non-Sudakov form factor acts only if $k_{\perp}>q_{i}$. We obtain the following $\Delta_{n s}$ form factors:

$$
\begin{aligned}
& \widetilde{\Delta}_{n s} \rightarrow \Delta_{n s}=\exp \left(-\int_{q_{i}}^{k_{\perp}} \frac{d q^{\prime 2}}{q^{\prime 2}} \int^{z M} d z \frac{1}{z}\right) \text { for } k_{\perp}>q_{i} \\
& \widetilde{\Delta}_{n s} \rightarrow \Delta_{n s}=1 \text { for } k_{\perp}<q_{i}
\end{aligned}
$$


In Fig. 3 the gluon density is shown as a function of $x$ for the evolution scale $\mu^{2}=100 \mathrm{GeV}^{2}$ using the benchmark starting distribution with a black curve. QCDNUM [13] agrees with PB method in

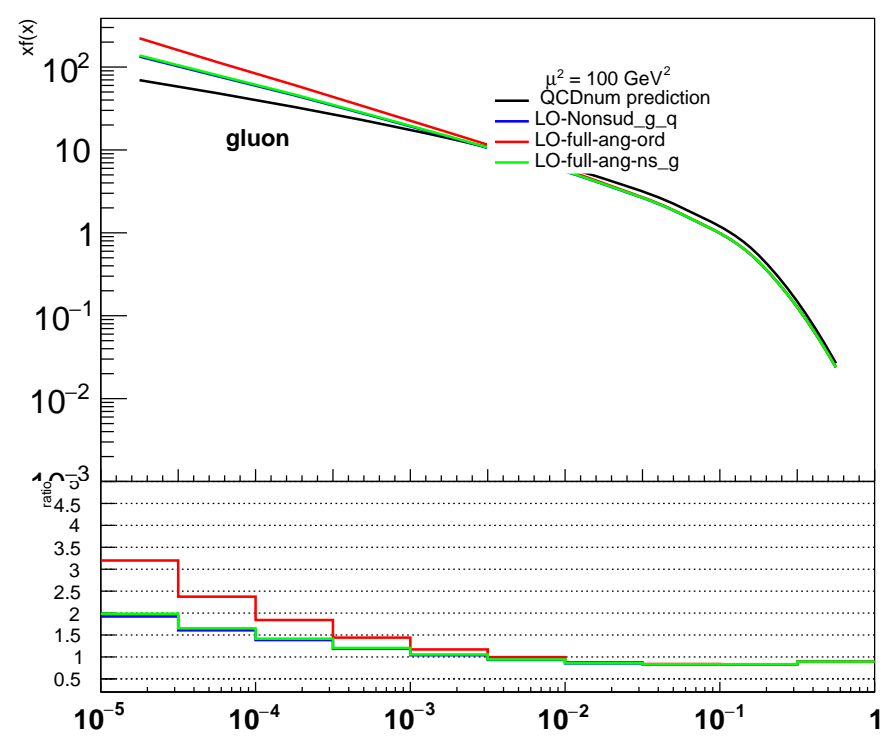

Figure 3: The black curve represents the QCDNUM prediction. The red curve includes full angular ordering. The non-Sudakov form factors are included in $p_{g g}$ and $p_{g q}$ in the green and blue curves, respectively.

the DGLAP limit as shown in ref. [1]. The red curve shows the results when the phase space is opened up to include angular ordering. It allows for unordered emission and increases the gluon density at small $x$, essentially below $10^{-3}$. Including virtual corrections via non-Sudakov form factor (for $k_{\perp}>q_{i}$ ) into $P_{g g}$ leads to small $x$ suppression (green curve in Fig. 3). Including the non-Sudakov form factor also for $P_{g q}$ leads to the blue curve which is slightly below the green one. However, the gluon density at small $x$ is still larger compared to DGLAP based curve.

This step by step study is possible via the PB method because it can solve the evolution equation via an iterative procedure.

\section{Conclusion}

TMD formalisms are relevant in QCD to describe contributions to the initial states of hadronic collisions both for high $x$ and for low $x$ (see e.g. [14]). An essential ingredient is provided by parameterizations and fits to experimental data for TMD distribution functions [15].

The PB method has been recently proposed as a flexible approach which can be applied to a wide range of processes and observables.

In this article we have performed new PB TMD fits at LO, and we have presented a method to incorporate CCFM effects into the PB formulation. Our results can be used to extend the existing simulations of QCD cascades into the semi-hard regime. 


\section{Acknowledgment}

STM thanks the Humboldt Foundation for the Georg Forster research fellowship.

\section{References}

[1] F. Hautmann, H. Jung, A. Lelek, V. Radescu and R. Zlebcik, JHEP 1801, 070 (2018) doi:10.1007/JHEP01(2018)070 [arXiv:1708.03279 [hep-ph]]

[2] F. Hautmann, H. Jung, A. Lelek, V. Radescu and R. Zlebcik, Phys. Lett. B 772, 446 (2017) doi:10.1016/j.physletb.2017.07.005 [arXiv:1704.01757 [hep-ph]]

[3] M. Ciafaloni, Nucl. Phys. B296 (1988) 49.

[4] S. Catani, F. Fiorani and G. Marchesini, Nucl. Phys. B 336, 18 (1990). doi:10.1016/0550-3213(90)90342-B

[5] F. Hautmann and H. Jung, JHEP 0810 (2008) 113.

[6] H. Abramowicz et al. [H1 and ZEUS Collaborations], Eur. Phys. J. C 75 (2015) no.12, 580 doi:10.1140/epjc/s10052-015-3710-4 [arXiv:1506.06042 [hep-ex]]

[7] R. K. Ellis, W. J. Stirling and B. R. Webber, QCD and collider physics, CUP 1996.

[8] A. Bermudez Martinez, P. Connor, F. Hautmann, H. Jung, A. Lelek, V. Radescu and R. Zlebcik, Phys. Rev. D 99, 074008 (2019) doi:10.1103/PhysRevD.99.074008 [arXiv:1804.11152 [hep-ph]]

[9] A. Bermudez Martinez et al., arXiv:1906.00919 [hep-ph].

[10] S. Alekhin et al., Eur. Phys. J. C 75 (2015) 304.

[11] F. Hautmann, H. Jung and S. Taheri Monfared, Eur. Phys. J. C 74 (2014) 3082.

[12] F. Hautmann and H. Jung, Nucl. Phys. B883 (2014) 1; arXiv:1206.1796 [hep-ph].

[13] M. Botje, Comput. Phys. Commun. 182, 490 (2011) doi:10.1016/j.cpc.2010.10.020 [arXiv:1005.1481 [hep-ph]]

[14] R. Angeles-Martinez et al., Acta Phys. Polon. B 46 (2015) 2501.

[15] F. Hautmann, H. Jung, M. Krämer, P. J. Mulders, E. R. Nocera, T. C. Rogers and A. Signori, Eur. Phys. J. C 74 (2014) 3220. 